\title{
Péritriches épizoïques dans la chambre branchiale des Balanidae (Crustacea : Cirripedia) \\ Epistylis Nigrellii n.sp. E. borizontalis (Chatton 1930)
}

\author{
par L. ARVY, A. BATISSE et D. LACOMBE ${ }^{1}$
}

(Laboratoire d'Histoenzymologie de la Faculté de Médecine de Paris, 45, rue des Saint-Pères F 75 - Paris-6 ${ }^{\circ}$ et Laboratoire de l'Evolution, 105, boulevard Raspail, F 75 -Paris-6 ${ }^{\circ}$ et Osborn laboratories of marine sciences, à New-York)

\section{Résumé}

La cavité branchiale de Balanus balanoides L, à New York, est très souvent parasitée par des Epistylis horizontalis Chatton (1936), de même que la cavité branchiale de B. eburneus contient des Epistylis Nigrellii n. sp.; ces deux espèces de Péritriches épizoiques sont décrites.

\section{Summary}

The branchial cavity of Balanus balanoides from New York harbor are very often the home of Epistylis horizontalis Chatton (1936), just as the branchial cavity of $B$, eburneus in the same place or at New Jersey, shelters Epistylis Nigrellii n. sp.; these two species of epizoic Peritriches are described.

(1) Nous adressons nos très vifs remerciements à Mlle de Villeroché qui a assumé la dactylographie de ce travail, et à M. Bernier qui a tiré de nos négatifs les meilleures photos possibles. 
Depuis l'observation d'Ehrenberg (1838) sur le Leucophrys anodontae, commensal dans la cavité palléale de l'Anodonte, de nombreux Ciliés ont été décrits, à diverses reprises dans les cavités palléale ou branchiale de divers Invertébrés, surtout chez les Mollusques (Fenchel, 1965). Il s'agit presque toujours de Thigmotrichida, d'Heterotrichida ou d'Hypotrichida, plus rarement de Peritrichida, essentiellement de la famille des Urceolariidae. Chez les Crustacés, la faune des Ciliés Péritriches habitant les cavités branchia'es est plus variée et comporte de nombreux représentants des familles Lagenophryidae, Vaginicolidae, Epistilydidae et Vorticellidae. Cependant, comme en témoigne le tableau ci-joint, les Epistylis inféodés à ce milieu particulier, sont relativement rares : on n'en compte que onze espèces (1) sur 85 espèces connues.

Il semble que le premier Epistylis décrit chez Balanus l'ait été par Merejkowski (1877) ; c'est l'Epistylis ba!anorum des branchies des B. balanoides de la Mer Blanche. Ensuite, il faut attendre jusqu'à 1930 et 1936, pour voir Chatton signaler que lorsqu'il déplissait avec soin sous le microscope, une mue de Balane, il voyait des Epistylis fixés sur la région de la «mue qui revêtait la cavité palléale du Crustacé », c'est-à-dire sa cavité branchia'e ; et cela aussi bien chez les Balanus eburneus de Woods-Hole (U.S.A.) que chez les Balanus amphitrite de l'étang de Thau (France). Ces Epistylis ne répondant exactement à aucune des formes révisées par Kahl (1934-5), il les dénomma Epistylis horizontalis. En 1947, Raabe retrouve Epistylis horizontalis sur les branchies des Balanus balanoides de la Baltique polonaise et plus récemment Jankowski (1967) observe ce même Péritriche dans les cavités branchiales des B. balanoides de la mer de Barents.

De nombreuses coupes histologiques et des dissections encore plus nombreuses, de divers Balanidae, faites en vue de l'étude enzymologique de leur « appareil cémentaire (Arvy et Lacombe, 1968) nous ont permis d'observer plusieurs parasites (Cephaloidophora communis, Pyxinioides bolitoides, Stylochus ellipticus, métacercaires d'un Microphallidae etc...) déjà décrits (Arvy et Nigrelli 1968) et, en outre, sur les branchies, de nombreux ciliés en cloche renversée, fixés sur un pédoncule ramifié, non contractile, Cilié Péritriches à l'évidence, objets de cette communication. Des Ciliés différents de ces Péritriches coexistaient parfois, mais ils étaient infiniment plus rares, de sorte que leur étude reste à faire.

Les Péritriches épizoïques dans la cavité branchiale existaient chez les Balanus eburneus Gould de New-Jersey * et de New-York. Ils étaient relativement peu nombreux, par comparaison avec ceux qui vivaient en commensaux, dans les branchies de Balanus balanoides L. Ces derniers existaient constamment, en juillet-août, chez les Balanes récoltés à marée basse, sur les jetées du Port de New-York, au voisinage des laboratoires Osborn (Coney Is!and).

Dans tous les cas, les Péritriches étaient apparemment inoffensifs pour le Balane car, à leur voisinage, les branchies n'étaient ni érodées, ni pigmentées, ni amincies, ni épaissies.

(1) Epistylis astaci, balanorum, bimarginata, cambari, carcini, epistyliformis, horizontalis, jaerae, ovalis, stammeri et thienemanni. Balanus.

Nous tenons à remercier encore M. Townshen, J. Smith qui a bien voulu nous procurer ces 


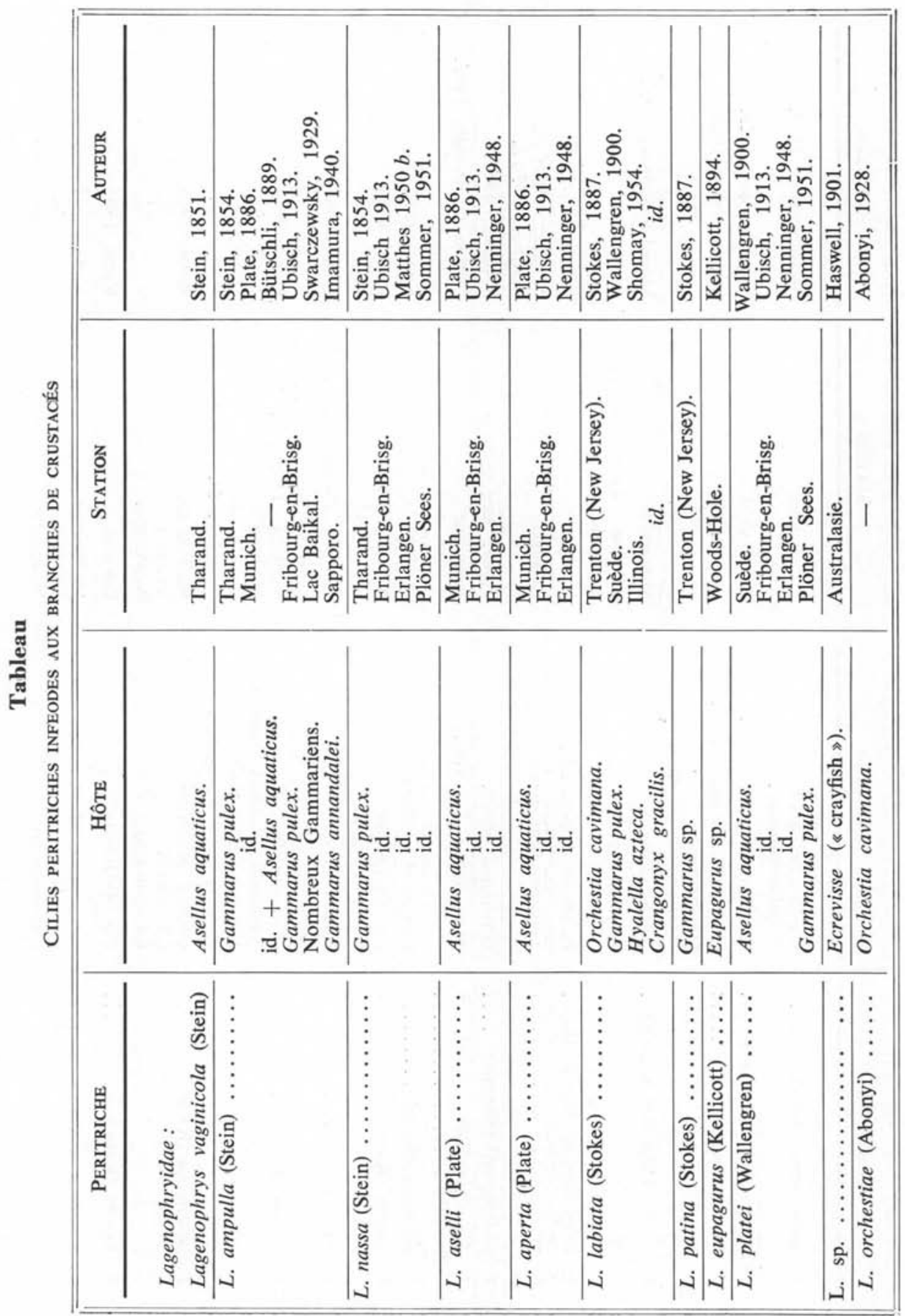



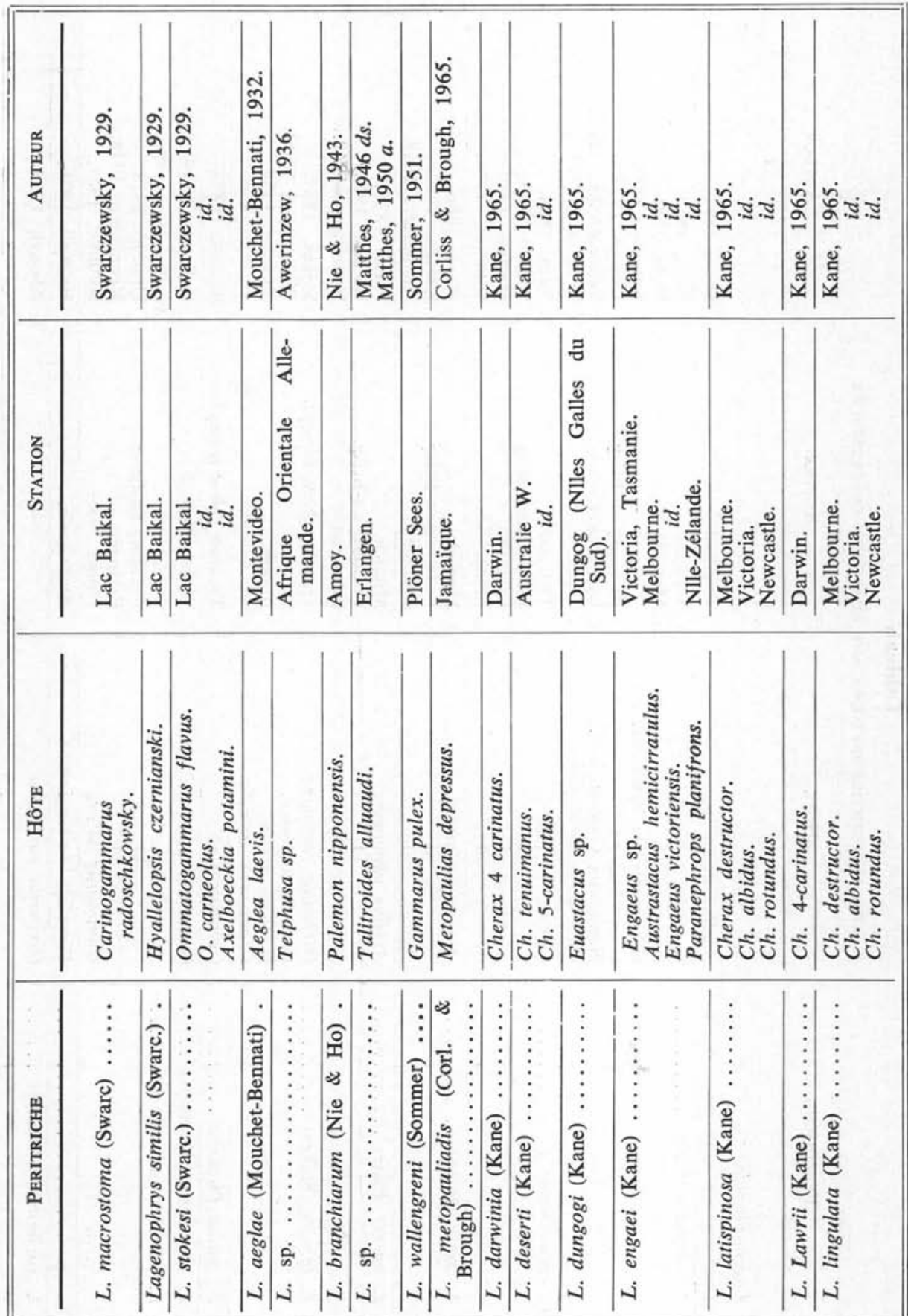

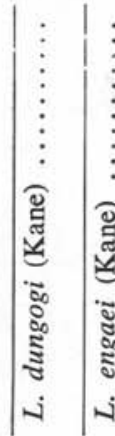

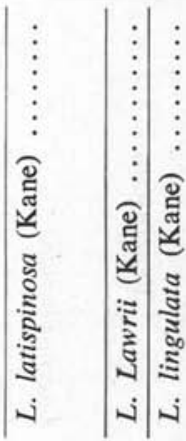




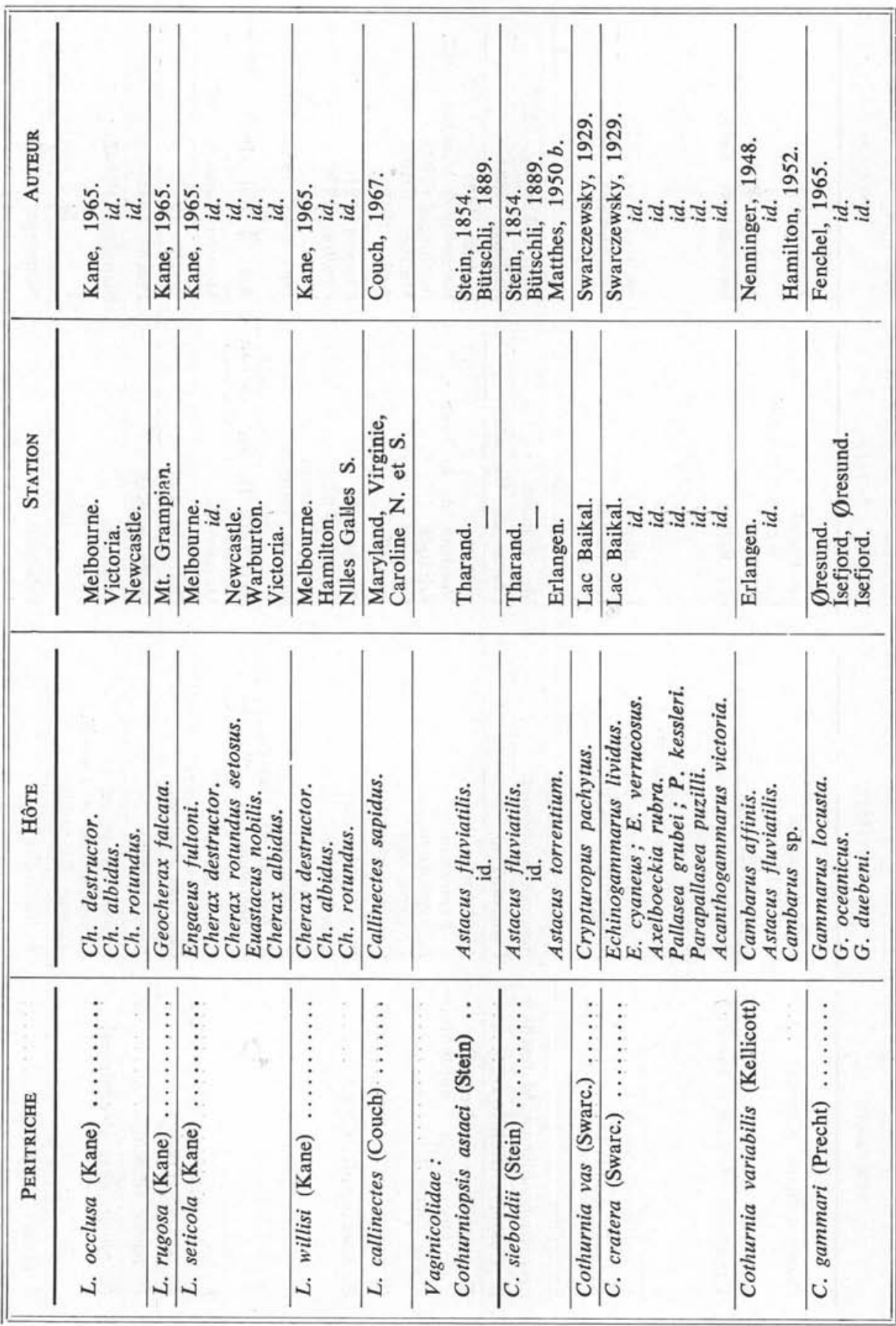



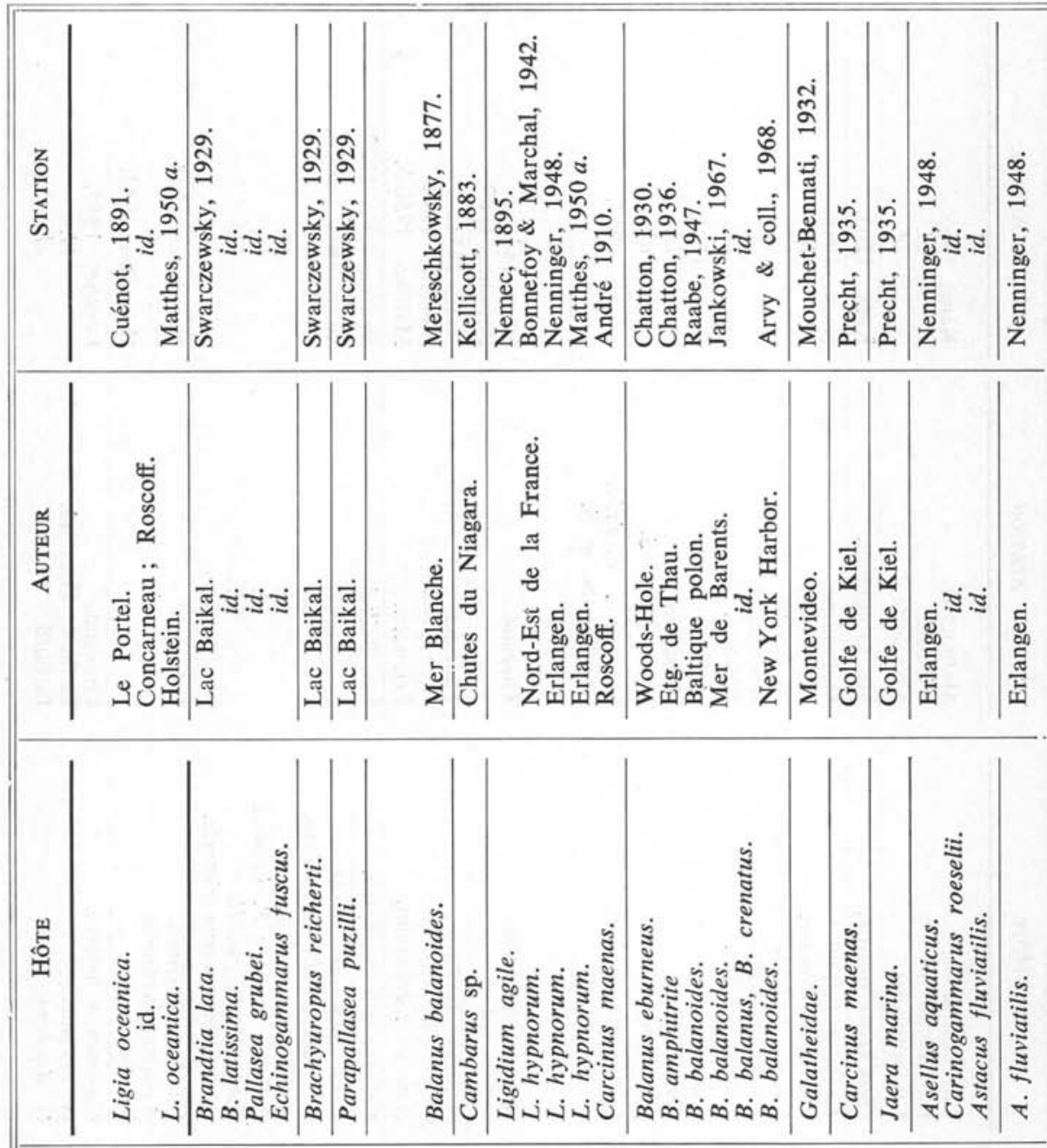

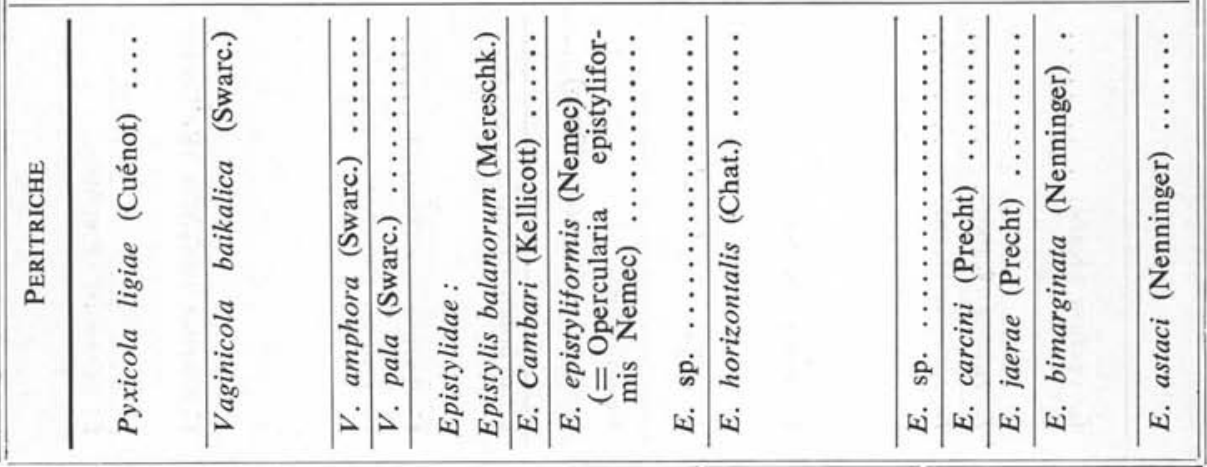




\begin{tabular}{|c|c|c|c|c|c|c|c|c|c|c|c|c|c|c|}
\hline$\frac{5}{3}$ & 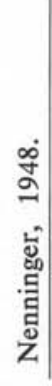 & 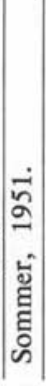 & 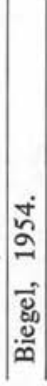 & 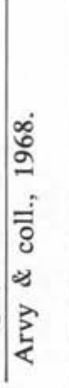 & 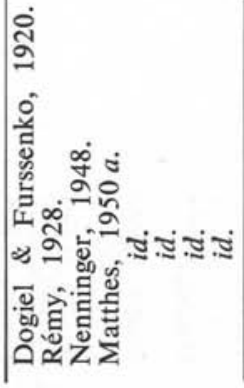 & 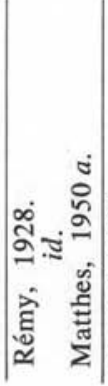 & 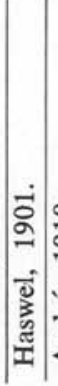 & 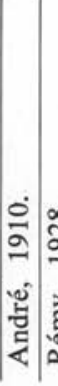 & 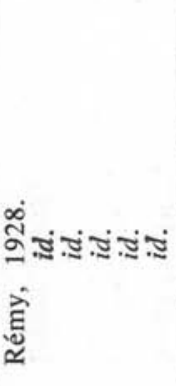 & 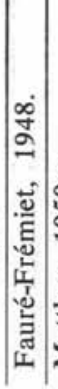 & 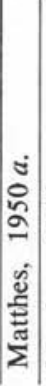 & 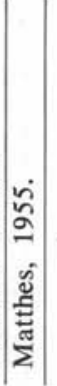 & 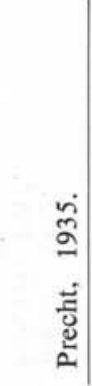 & 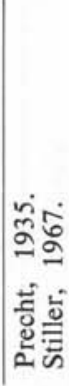 \\
\hline$\underset{\substack{z \\
\vdots}}{\stackrel{z}{5}}$ & 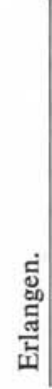 & 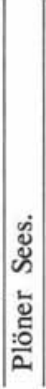 & 离 & 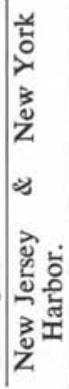 & 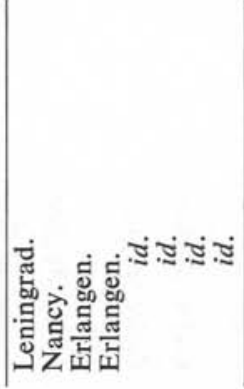 & 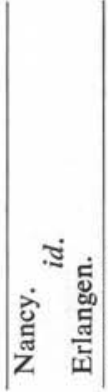 & 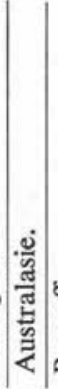 & 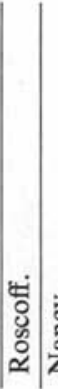 & 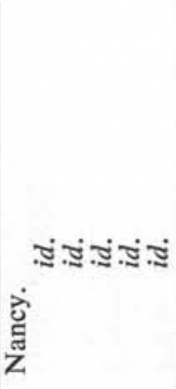 & 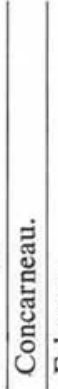 & 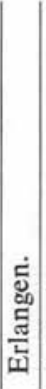 & 总 & 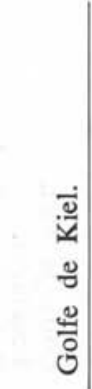 & 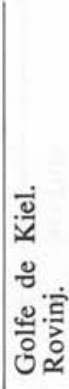 \\
\hline 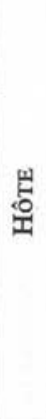 & 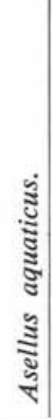 & 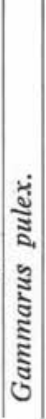 & 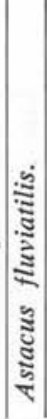 & 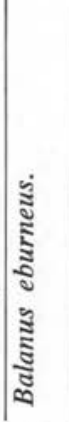 & 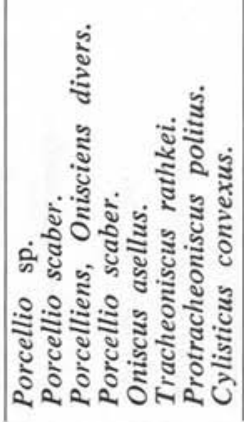 & 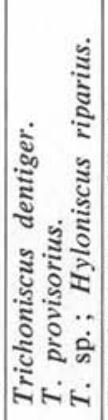 & 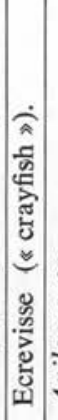 & 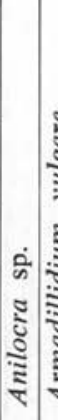 & 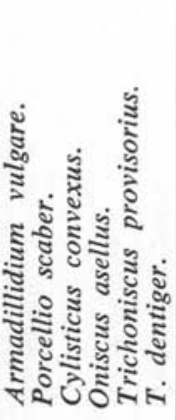 & 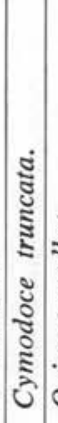 & 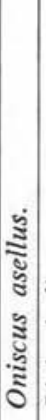 & 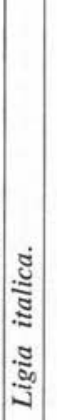 & 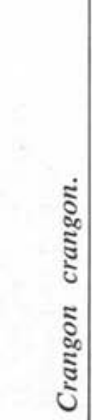 & 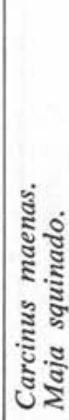 \\
\hline 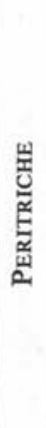 & 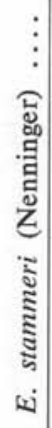 & 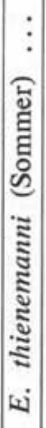 & 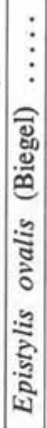 & 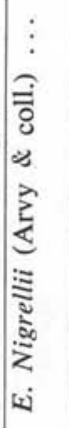 & 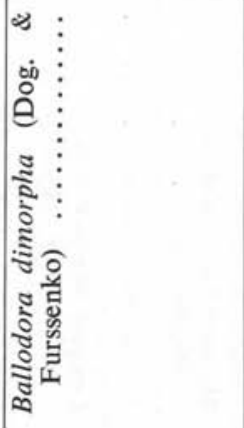 & 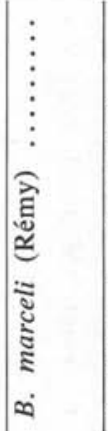 & 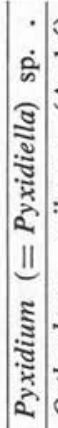 & 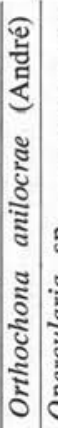 & 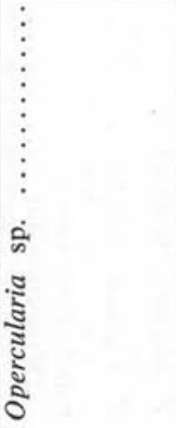 & 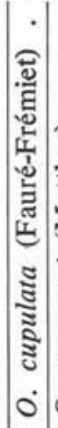 & 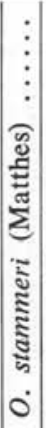 & 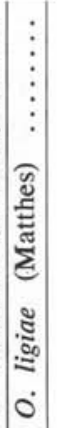 & 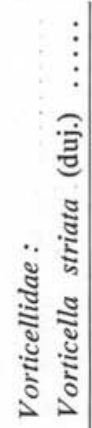 & 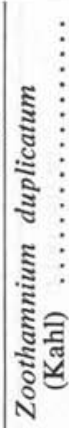 \\
\hline
\end{tabular}




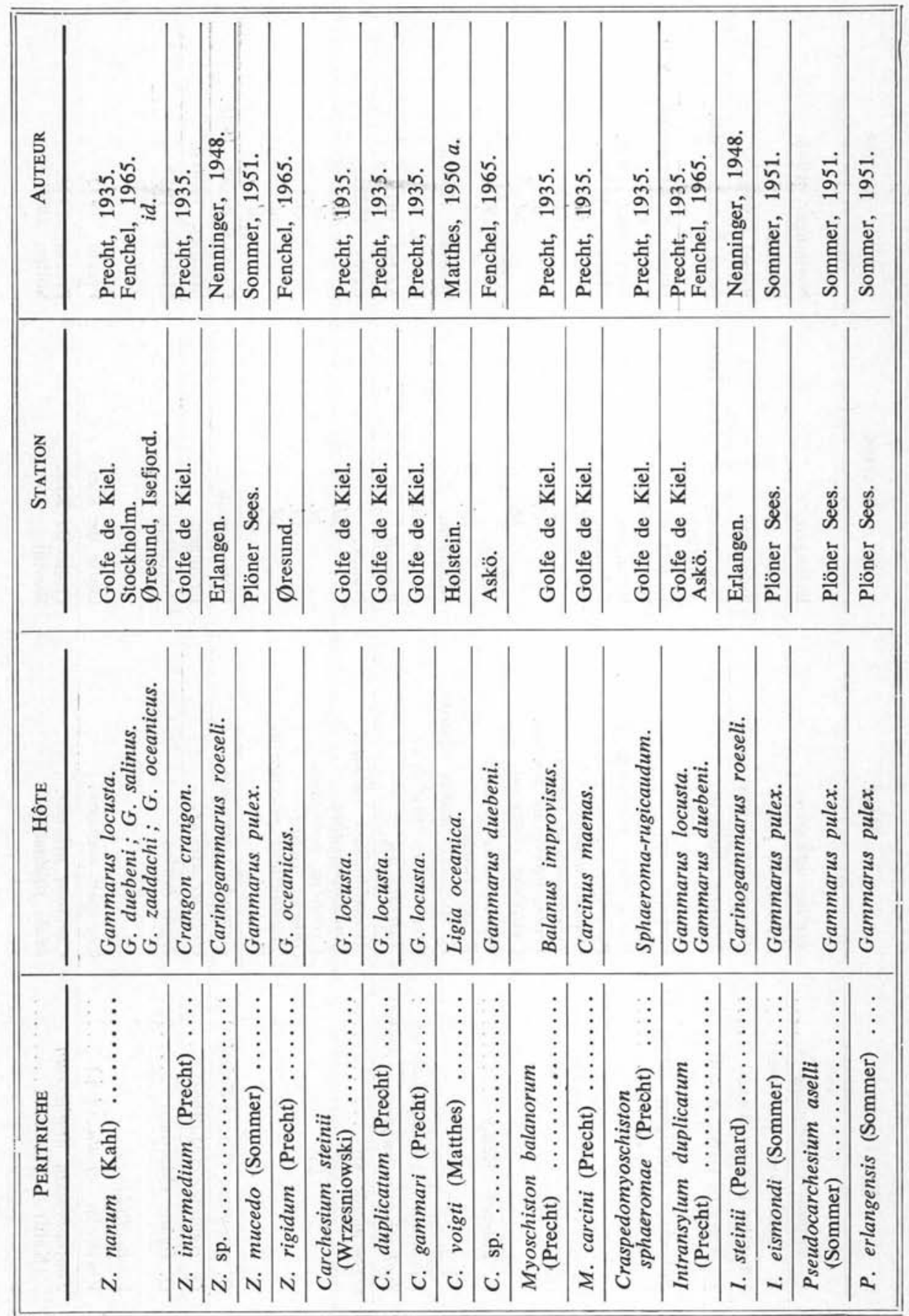


Il s'agissait à l'évidence de deux Péritriches différents, le parasite de $B$. eburneus étant généralement beaucoup plus petit que celui de $B$. balanoides, ce dernier étant très proche, sinon identique à l'Epistylis horizontalis Chatton.

Des recherches bibliographiques approfondies nous ont rapidement convaincus que l'un au moins de ces deux Péritriches était nouveau et le protistologue de notre équipe s'est spécialement attaché à réaliser sur ces deux Péritriches l'imprégnation argentique, pratique indispensable à la compréhension des structures buccales, à la réalisation d'images synthétiques (fig. 1 et 2 ) et à la distinction de deux espèces voisines comme l'ont montré tous les travaux exécutés sur ce thème depuis Chatton et Lwoff (1930) et pius particulièrement, les travaux de Dragesco (1962), Lom (1961-4) et Tuffrau (1967). Cette recherche nous a permis de caractériser les deux espèces d'Epistylis vivant dans la cavité branchiale des Balanus que nous avons examinés; il nous paraît indubitable que l'Epistylis de Balanus eburneus Gould est inconnu; nous le décrivons donc, en le dédiant au Docteur Nigrelli, Directeur du Laboratoire Osborn, lieu où cet Epistylis a été découvert. Tout un ensemble d'observations obtenues après mise en œuvre de techniques variées nous permettent de définir les deux espèces d'Epistylis que nous avons étudiées chez Balanus.

\section{Matériel et Méthodes.}

L'ensemble des observations a comporté d'abord l'examen des Ciliés in vivo, sur des branchies disséquées, prélevées sur des Balanus balanoides L. du port de New-York et sur des Balanus eburneus Gould du même biotope, ou du New-Jersey. En outre, des Balanus, fraîchement récoltés, ont été fixés, en vue de l'étude histologique dans différents fixateurs: alcool à $95^{\circ}$, formol à $10 \%$, Susa, Bouin, Carnoy. Après inclusion à la paraffine, les tissus coupés à $7 \mu$ ont été colorés par l'hématoxyline de Heidenhain, l'azan de Heidenhain, le Kernechtrot, le bleu alcian, le vert naphtol etc... En outre, des Epistylis récoltés sur les branchies de Ba'anus fixés in toto ont été imprégnés par le protéinate d'argent, suivant la technique de Bodian, modifiée par Dragesco (1962) et Tuffrau (1967). Après rinçage par l'eau distillée, les Epistylis ont été fixés sur lames à l'aide d'albumine glycérinée de Mayer, avec léger chauffage et tannage par le mélange classique alcool-formol. Après rinçage, les parasites ont été exposés au protargol (à $1 \%$ dans l'eau distillée) pendant 48 heures, à froid. Après révélation hydroquinonique et virage au chlorure d'or-acide oxalique, la réaction argentique a été fixée par l'hyposulfite de sodium et les préparations ont finalement été déshydratées et montées (PI. I et III).

\section{Observations.}

$1^{\circ}$ Epistylis Nigrellii, n.sp. des branchies de Balanus eburneus Gould, de New Jersey et de New-York (fig. 1 A-B).

La forme sédentaire est coloniale, chaque individu occupant l'extrémité d'une tige ramifiée (fig. $1 \mathrm{~A}$ ). En expansion, les individus sont campanulaires ; contractés, ils sont 

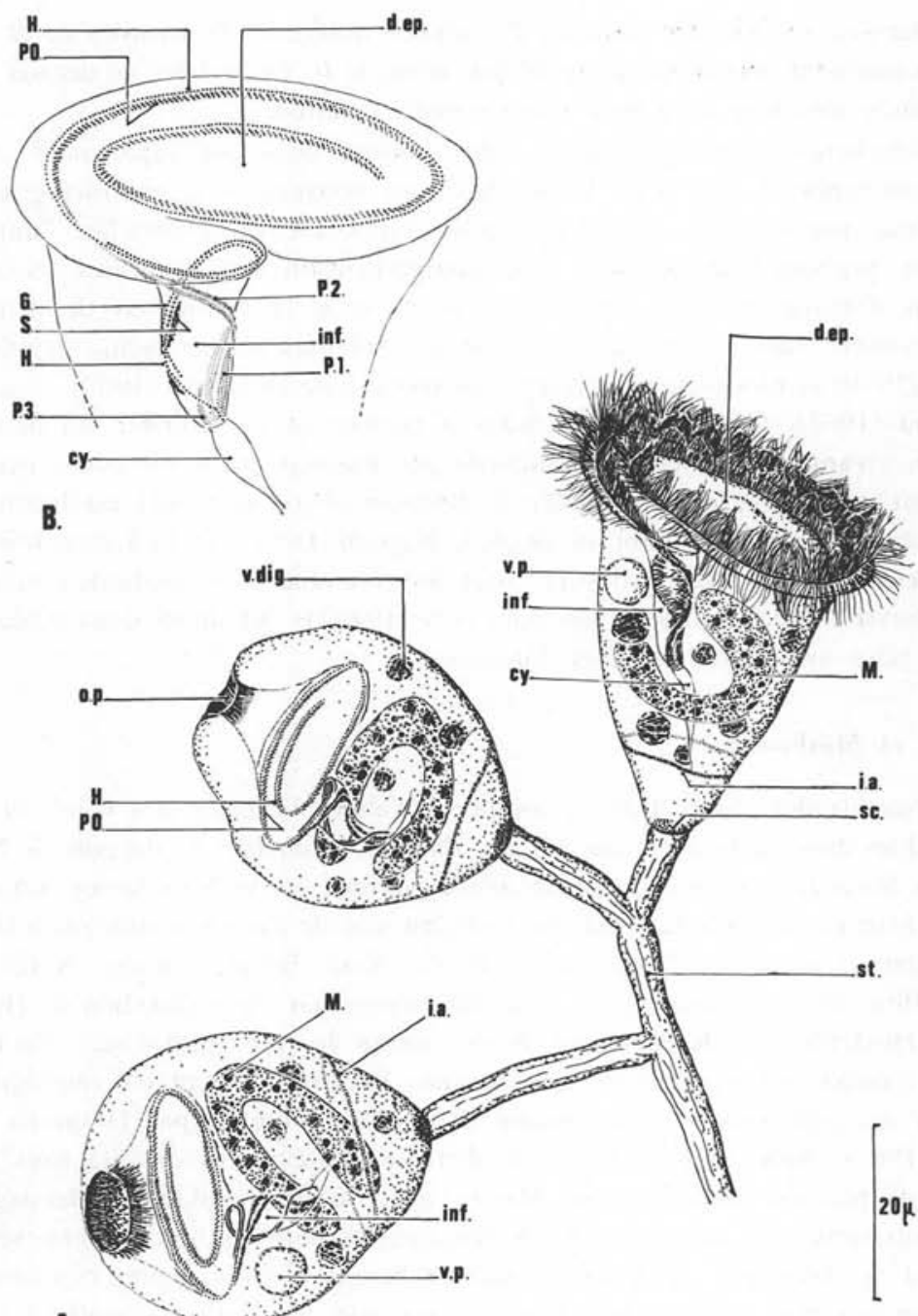

A.

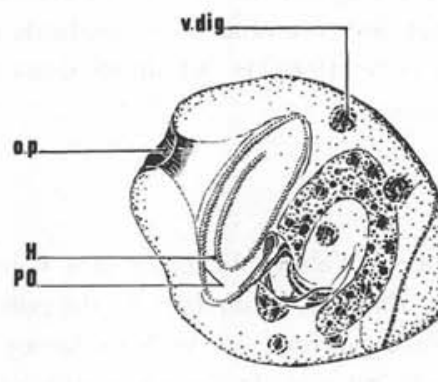


ovoïdes. Ils apparaissent très généralement un peu plus longs que larges (35 à $40 \mu$ de long, pour $33 \mu$ de large, par exemple). Le style s'élargit au voisinage de la scopula pour atteindre $4 \mu 3$. Il est exceptionnel de trouver des individus plus gros, atteignant 45 et même $50 \mu$ de long.

L'infraciliature aborale (i.a.) est formée d'un anneau fermé, épais, constituée très globalement par trois rangs de cinétosomes serrés.

L'infraciliature du disque épistomien dessine une spirale enroulée dans le sens anthoraire, décrivant un tour trois-quart au moins, et comportant l'ensemble classique de la polycinétie PO (trois rangs de cinétosomes coalescents) et de l'haplocinétie $\mathrm{H}$ (un rang apparent de cinétosomes). Les cils longs et flexueux, implantés sur ces cinéties, forment deux «membranes » internes (correspondant à PO) et une externe (portée par $\mathrm{H}$ ). Au terme de sa course sur le disque épistomien, PO plonge directement à partir du côté externe de l'ouverture buccale, dans le tube infundibulaire. Elle en suit la paroi selon un parcours spiral d'un tour, jusqu'au cytostome, tout en se divisant en deux, puis trois peniculus parallèles (fig. $1 \mathrm{~B}$ ). $\mathrm{H}$, au contraire suit le bord de l'entrée buccale selon un demi-tour avant de descendre le long de la paroi infundibulaire, où elle décrit un parcours spiral d'un tour, jusqu'au cytostome. Elle est donc, dans l'infundibulum, toujours décalée de $180^{\circ}$ par rapport à PO. Une cinétie «ger minale » $G$ (cinétie stomatogène) accompagne $H$, depuis l'entrée péristomienne jusqu'à mi-longueur du tube péristomien à un niveau où une structure argyrophile $\mathrm{S}$ peut être décelée à leur voisinage immédiat.

Le macronuoleus forme un fer-à-cheval autour du tube infundibulaire; d'affinité tinctoriale uniforme, après coloration, par le kernechtrot, il apparaît parfois plurinucléolé et l'hématoxyline au fer, comme l'imprégnation argentique, y révèle plusieurs masses sphériques; elles sont très argyrophiles et réparties dans une matrice finement granuleuse.

En somme, les caractères majeurs, permettant la diagnose d'Epistylis Nigrellii $\mathrm{n}$. sp. sont les suivants : Epistylis assez rare, formant à l'état sédentaire, de petites colonies (fig. $1 \mathrm{~A}$ et 4 ) ; d'environ six individus transparents, à zoïdes unis par des rameaux stylaires, faiblement striés intérieurement par des fibrilles longitudinales, et légèrement plissés superficiellement. Le corps est campanulaire. La ciliature buccale est composée d'un ensemble haptocinétie/polycinétie décrivant sur le disque épistomien un tour trois-quart. La polycinétie décrit un tour dans le tube infundibulaire (fig. $1 \mathrm{~B}$ ) et s'y divise en trois peniculus parallèles entre eux, le troisième étant très court et situé près du cytostome. La cinétie germinale $(\mathrm{G})$ est relativement longue, puisqu'elle accompagne l'haplocinétie depuis l'entrée infundibulaire jusqu'à mi-profondeur. Le macronucleus est pourvu d'amas argyrophi'es, dans un contenu fondamental finement granuleux.

Dimensions : Un peu plus long que large : 35 à $40 \mu$ de long pour $33 \mu$ de large ; n'atteint qu'exceptionnellement $50 \mu$ de long.

Hôte: Balanus eburneus Gould. Fixé sur les lamelles branchiales et jusqu'à la base des cirres.

Stations : New-Jersey et New-York. 
$2^{\circ}$ Epistylis horizontalis, Chatton (1930 et 1936), des branchies de Balanus balanoides L, de New-York Harbor (fig. 2, A-B).

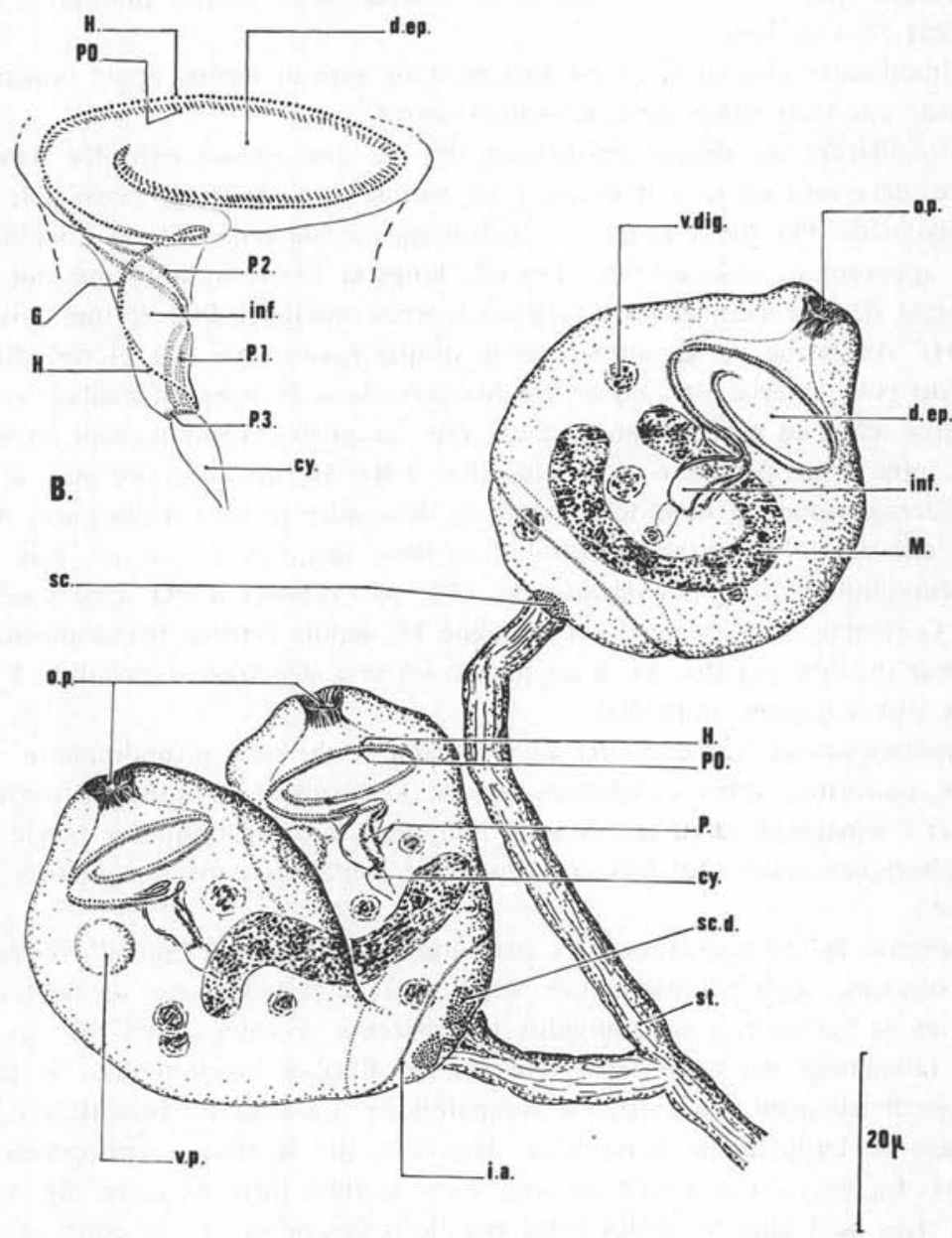

A.

Fig. 2. - Epistylis horizontalis Chatton, des branchies de Balanus balanoides L., de New-York.

$\mathrm{P}_{1}, \mathrm{P}_{2}, \mathrm{P}_{3}=$ penicu'us prolongeant la polycinétie dans l'infundibulum.

$P O$. $=$ polycinétie.

$S .=$ structure argyrophile infundibulaire.

sc. $=$ scopula.

sc. $d .=$ scopula en cours de division.

st. $=$ rameaux stylaires unissant les zoïdes de la co'onie.

$v$. dig. $=$ vacuoles digestives.

v.p. $=$ vésicule pulsatile. 


\section{A) Forme sessile :}

La grande abondance de ces Péritriches nous a permis d'en faire une étude assez complète. Sur les coupes histologiques passant par l'axe de l'animal, ce dernier apparaît fixé sur un style assez long et grêle. Le corps est campanulaire (fig. 12, 13, 15) et le pôle apical est abondamment cilié. Certains Péritriches, coupés transversalement ou obliquement, appartiennent à l'évidence à la même espèce (fig. 14).

Il existe, en outre, des Infusoires infiniment plus plats (fig. 17, 18) libres entre les lamelles branchiales, patelliformes, qui sont restés énigmatiques jusqu'à la découverte du travail de Chatton (1936).

Avec la connaissance de ce dernier, tout mystère a disparu. En effet, en observant des exuvies de Balanes à Woods-Hole, en 1930, Chatton remarqua des Ciliés très aplatis dorso-ventralement, porteurs sur tout leur pourtour d'une épaisse frange ciliaire, fermée en un cercle complet au fond d'un profond sillon. Ces Ciliés avaient un péristome contracté et, sur leur face inférieure, ils portaient une cicatricule correspondant à une scopula. Chatton les identifia comme des formes errantes, autonomes, caractérisées par leur asymétrie et leur hétéropolarité, dérivées par aplatissement, des Epistylis sédentaires à arbuscules rigides, sans spasmonèmes, coexistant dans les mêmes exuvies. Des observations répétées lui permirent, en effet, de percevoir la continuité entre les deux types d'organismes, les formes sédentaires évoluant en formes migratrices (fig. 3 ). Chatton (1936) a pu observer le déroulement de cette métamorphose en trois quarts d'heure environ chez des Epistylis placés entre lame et lamelle et examinés au microscope; il a vu les branches des arbuscules se couronner d'organismes aplatis, qui se détachaient et glissaient sur la chitine de la paroi de l'exuvie. Les migrateurs gardaient leur macronucleus, en large ruban, disposé en épingle à cheveux. En somme, le migrateur à grand axe horizontal résultait de l'évolution avec déformation de l'Epistylis fixé, de sorte que Chatton le dénomma horizontalis. Cette déformation (fig. 23) qui dessert le Cilié pour la nage, l'assujettit aux surfaces. La signification de cette évolution reste inconnue ; il est peu vraisemblable qu'elle réponde à un stade préparatorre à la multiplication des individus, car, en effet, Chatton (1936) qui a suivi la métamorphose du sédentaire en migrateur in vivo, admet que le premier signe de la transformation est la disparition des gastrioles, l'élimination des résidus alimentaires et la fermeture du péristome. Or, d'une part, les migrateurs n'ayant pas de gastrioles, ne se nourrissent pas et d'autre part, leur vésicule contractile ne fonctionne qu'à de rares intervalles. Ne se nourrissant pas, ils ne se divisent pas. En outre, des formes de conjugaison entre sédentaires existent, quoique rarissimes (fig. 19) et il existe des formes de scisșion, à clivage longitudinal (fig. 25,26 ). Il faut donc admettre que la métamorphose du sédentaire en migrateur correspond à un déterminisme inconnu, incomplètement lié au cycle d'exuviation de l'hôte, qui incite le futur migrateur à s'isoler et à choisir un autre gîte, où il se fixe et évolue en sédentaire colonial.

L'Epistylis sédentaire est pourvu d'un style non contractile, apparemment sans spasmonème * et sans structure, mise à part une faible striation au voisinage de la

* Rappelons que Chatton (1936) a observé un spasmonème réduit et partiel chez les $E$. horizontalis de l'étang de Thau. 

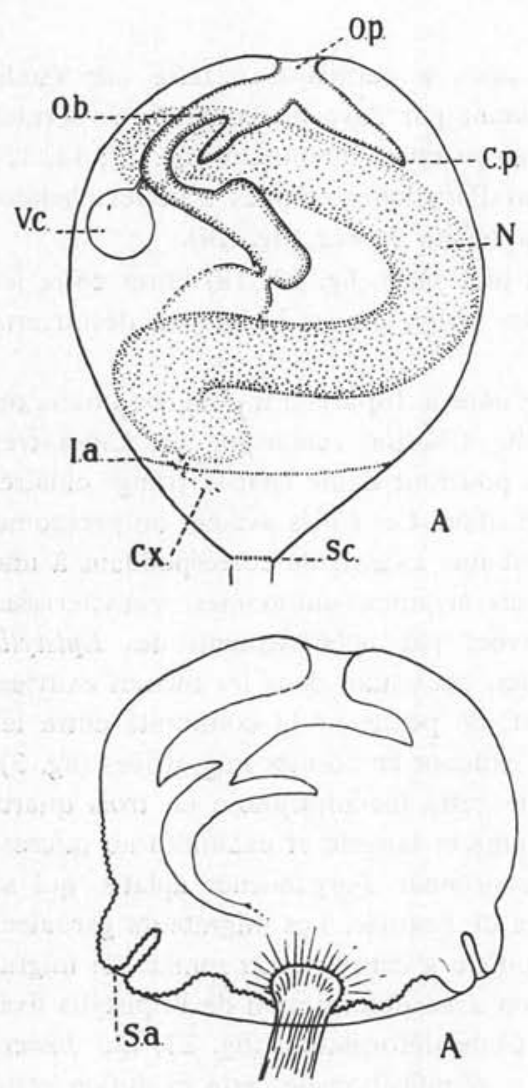

FIG. 3. - Schéma de l'organisation du sédentaire, vu de profil et des deux premiers stades de sa transformation en migrateur. O.p. = orifice du péristome ; C.p. = contour du péristome ; O.b. $=$ orifice buccal ; $\mathrm{Cx}=$ extrémité du cytopharynx ; $\mathrm{N}=$ macronucleus, tels que les a vus Chatton, en 1936

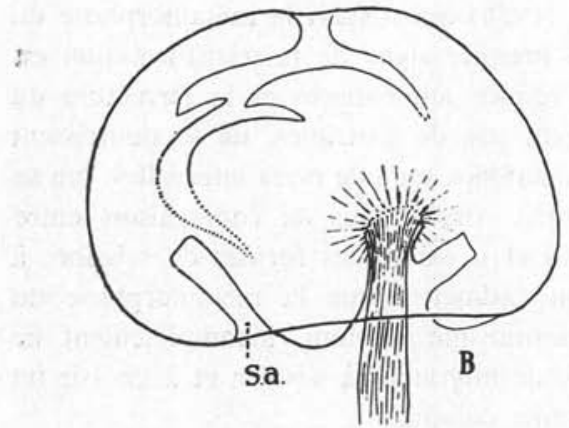

scopula et quelques menus plissements longitudinaux. L'infraciliature aborale dessine une ligne fermée, très mince, constituée par deux rangées de cinétosomes très rapprochés les uns des autres. L'infraciliature buccale (fig. 2 B) décrit un tour $1 / 8$ sur 

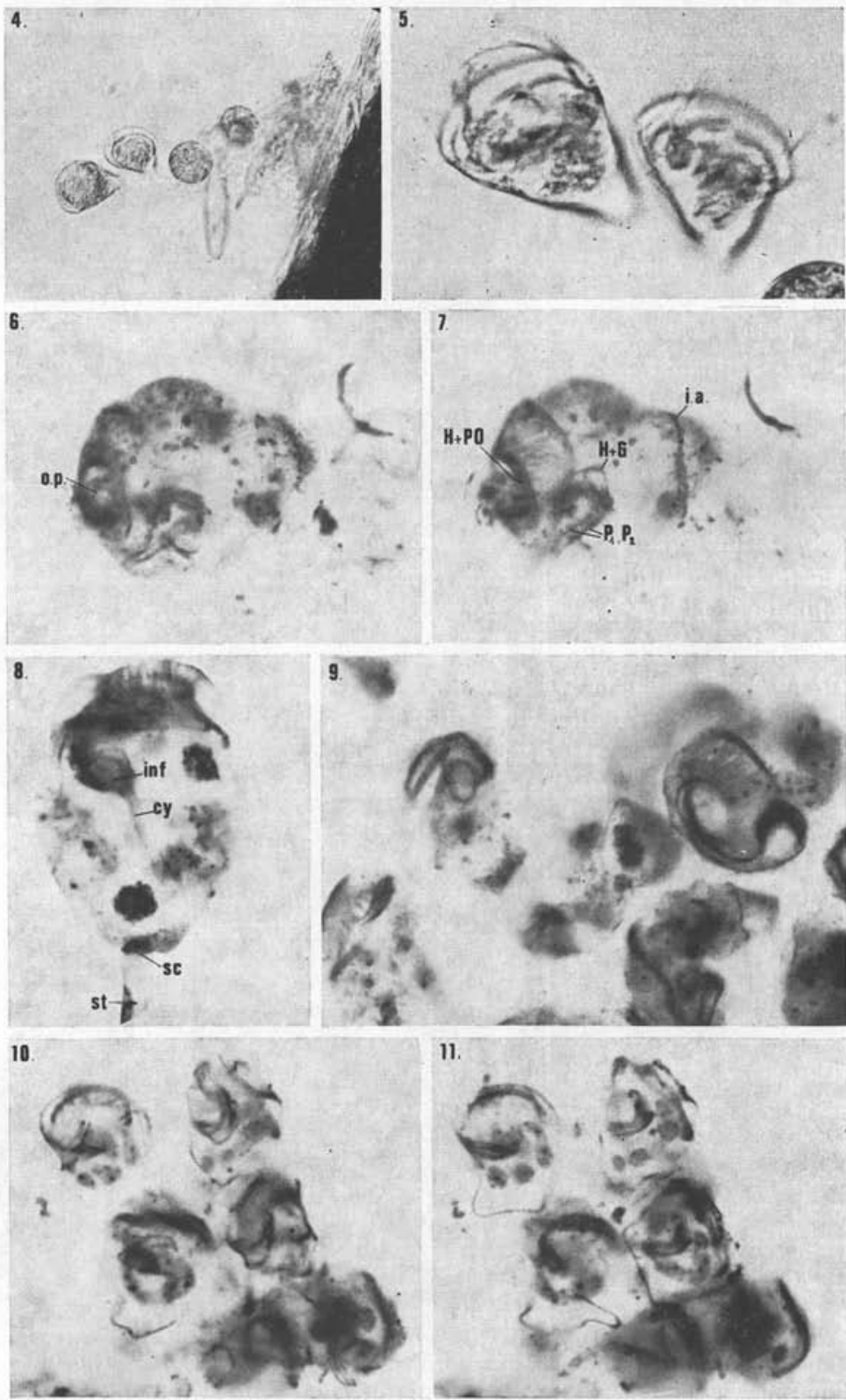

Fic. 4 et $5 .-$ Epistylis Nigrellii, vus in vivo ; Fig. $4: \times 250$; Fig. $5: \times 1000$

FiG. 6 à 11. - Epistylis Nigrellii, divers aspects, après imprégnation argentique. Fig. 6 et $7: \times 1100$. Fig. $8: \times 1100$. Fig. 9 à $11:$ colonies, $\times 1000$ 

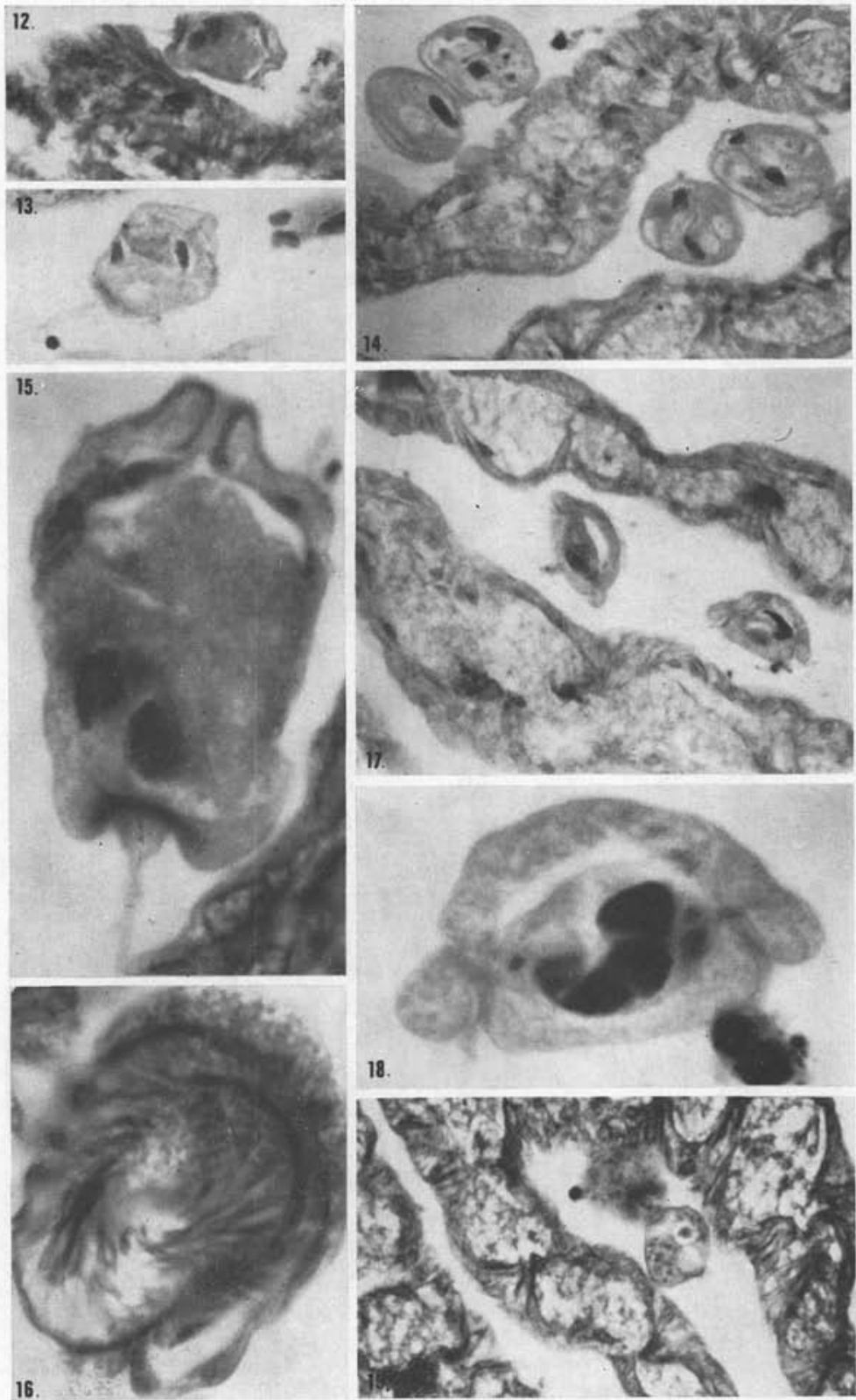

18.

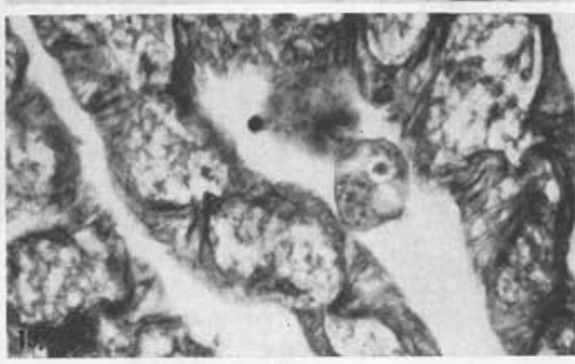

PI. II. - FIG. 12 à 19: Episty'is horizontalis Chatton; FIg. 12 et 13, coupes axiales, passant par le pédicule, après fixation par le liquide de Bouin et coloration par le kernechtrot-vert naphtol. Fig. 14: mêmes Epistylis, coupés transversalement. Fig. 15: Fort grossissement d'un individu coupé suivant son grand axe longitudinal. FIG. 16: vue apicale d'un Epistylis horizontalis Chatton, après coloration par l'hématoxyline au fer de Heidenhain, pour montrer la ciliature. Fig. 17 et 18 : faible et fort grossissement des migrateurs, après coloration par le kernechtrot-vert de naphtol. Fig. 19: aspect de la conjugaison, après fixation par le Carnoy et coloration par l'azan de Heidenhain. 

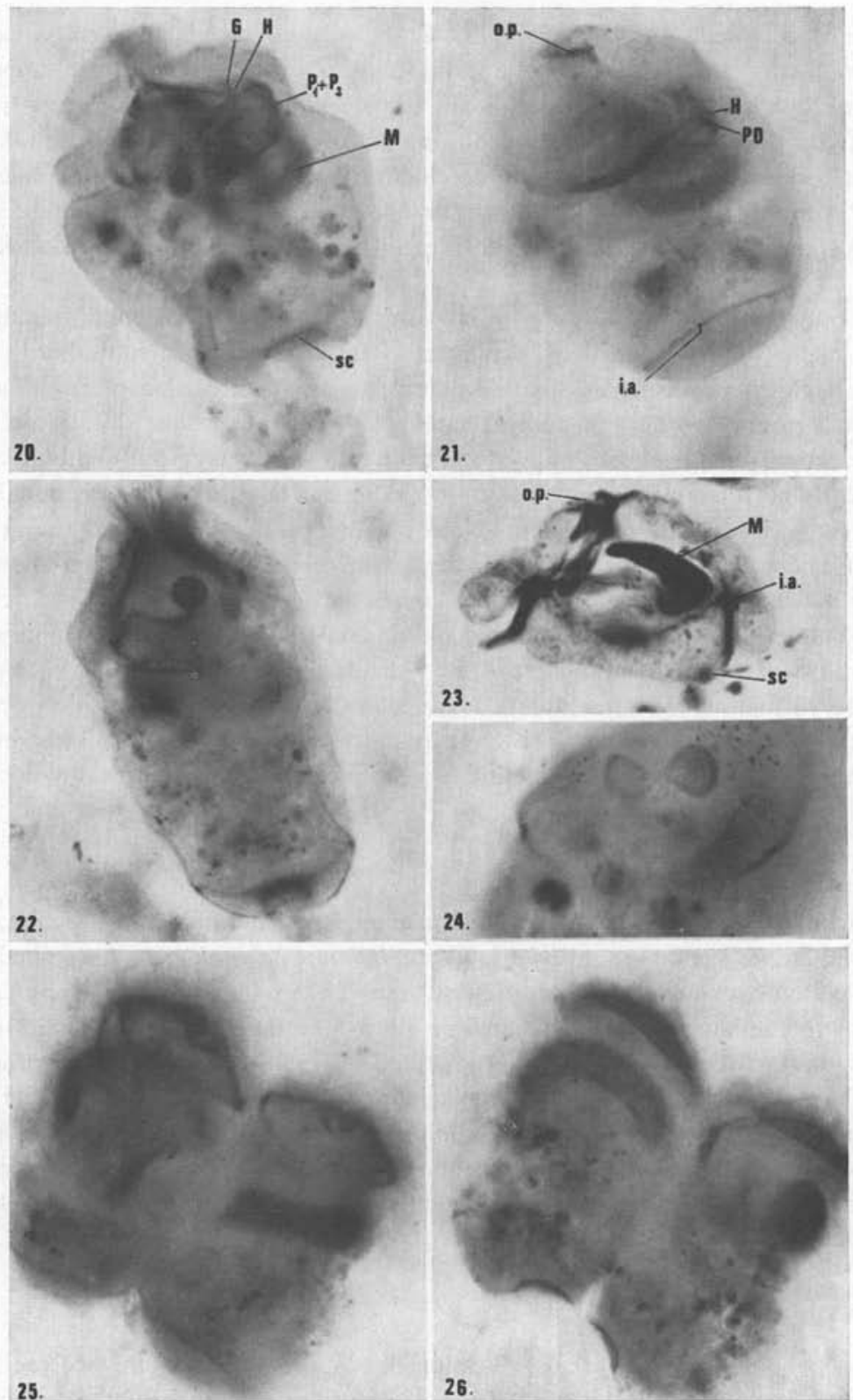

PI. III. - Fig. 20 à 26: Epistylis horizontalis Chatton, après fixation par le liquide de Bouin et imprégnation argentique. Fig. 20, 21 et 22, formes sédentaires; FIG. 23 : forme migratrice. Fig. 24,25 et 26, divers aspects de la division de cet Epistylis. Les lettres ont la même signification que pour les figures de texte 
le disque épistomien. La polycinétie PO a ses groupes de cinétosomes si rapprochés qu'elle apparaît comme une bande homogène d'aspect foncé, après imprégnation argentique; en réalité, elle est constituée par de petits traits obliques ou transverses (fig. 2 B) qui correspondent à des groupes de trois cinétosomes accolés. L'haplocinétie $\mathrm{H}$ est formée d'une rangée étroite de cinétosomes; cette rangée semble bien unique. Nous n'avons pu déceler sur les Epistylis que nous avons examinés un second rang de cinétosomes, non cilifères, analogue à celui découvert par Noirot-Timothée et Lóm (1965).

La polycinétie plonge le long de la paroi de l'infundibulum en décrivant un tour de spire ; elle s'y divise en deux peniculus et un troisième peniculus borde le cytostome. L'haplocinétie décrit un quart de cercle avant d'entrer dans l'infundibulum, où elle plonge en une spirale d'un tour; l'haplocinétie se trouve donc décalée de $180^{\circ}$ par rapport à la polycinétie, de sorte qu'elle se trouve sur la paroi de l'infundibulum opposée à celle de la polycinétie. L'haplocinétie est bordée par la strie germinale, depuis l'entrée péristomienne jusqu'à mi-profondeur. Nous n'avons pu déceler de structure argyrophile doublant l'haplocinétie dans l'infundibulum, telle que celle, décrite par Lom (1964). Le macronucleus est en forme de boudin courbé en fer à cheval, horizontal, centré par l'infundibulum; il est souvent plurinucléolé et contient des amas argyrophiles disséminés sur un fond finement granuleux. Une vésicule pulsatile est souvent visible près de la bouche infundibulaire et semble s'ouvrir dans cette cavité. En position de contraction (fig. 2 A), des ouvertures péristomiennes persistent ; elles apparaissent en sombre et finement striées après imprégnation argentique, grâce aux fibres myoïdes qui les bordent d'un véritable sphincter.

\section{B) Les migrateurs:}

Aucun individu de ce stade évolutif n'a pu être identifié à la dissection, ils n'ont été retrouvés que sur coupes histologiques de Balanus balanoides L. fixés par le Carnoy ou par le Bouin, et inclus à la paraffine. Comparés aux individus sédentaires, ces Epistylis sont très aplatis. Ils mesurent environ 46 à $50 \mu$ de long pour 25 à $29 \mu$ de haut. Ils sont pourvus d'une épaisse frange aborale; elle s'invagine dans un profond sillon. Le disque épistomien est également invaginé profondément dans une cavité à étroite ouverture entourée de myonèmes argyrophiles (fig. 23). Le macronucleus a les mêmes affinités tinctoriales que chez le sédentaire; il apparaît néanmoins souvent moins rubanné et plus massif. Vu de profil, nos migrateurs rappellent de très près les migrateurs décrits par Chatton en 1936.

\section{REPRODUCTION :}

Nous avons recherché avec application des aspects pouvant être interprétés comme des formes de conjugaison ou de division; ces formes sont rarissimes. Certains aspects (fig. 19) peuvent rationnellement être acceptés comme étant des images de conjugaisons et deux individus en division ont été observés ; ces derniers représentent le stade final de la division puisque la plasmodiérèse est presque achevée. Les deux jeux de cinéties 
buccales filles sont identiques et homothétiques (fig. 25, 26); à ce stade il n'est plus possible de voir la filiation originelle exacte de chaque cinétie, telle qu'elle est décrite par Chatton et Villeneuve (1937) ou par Lom (1964); le macronucleus est en intercinèse.

\section{Discussion.}

Nos observations appellent des remarques de divers ordres.

A) L'inocuité $d u$ parasitisme.

Les Péritriches commensaux des branchies des Cirripèdes sont, très vraisemblablement inoffensifs ; à l'abri des brusques variations de température et des prédateurs, ils tirent profit des courants brassés par les cirres et qui baignent les lamelles branchiales. Ils sont rejetés avec le revêtement cuticulaire branchial, à chaque mue et de même que les Crabes qui ont mué récemment n'ont pas de Lagenophrys (Couch, 1967), les Cirripèdes qui viennent de muer doivent en être dépourvus. On conçoit aisément, néanmoins, qu'une invasion massive de la cavité branchiale par des Epistylis puisse freiner les échanges du Crustacé avec le milieu où il respire. Mouchet (1932) a vu des Lagenophrys aeglae envahir, au cours de l'hiver, toute la surface branchiale d'Aeglea laevis. A cette époque, en effet, à Montévideo, ces Crustacés sont devenus adultes et ne muent pas; dans ce cas, la branchie réagit au voisinage immédiat des Péritriches, en élaborant un pigment brun ferrugineux, riche en peroxydase (Mouchet-Bennati, 1932) ; c'est là à notre connaissance la seule réaction du tissu branchial, qui ait été observée, sous l'effet de l'infestation par les Péritriches.

\section{B) La spécificité parasitaire.}

Cette spécificité semble bien réelle. Nos observations faites sur les $B$. balanoides et $B$. eburneus du port de New-York, révèlent que, prises dans le même biotope, les deux espèces de Cirripèdes, hébergent deux espèces d'Epistylis. De même, les Chthamalus de l'étang de Thau, ou de Banyuls, n'avaient pas les Epistylis vus chez des Balanus par Chatton (1936). De même encore, les Panopeus herbstii, les Eurypanopeus depressus et les Libinia emarginata qui vivent dans le même biotope que Metopaulias depressus, Crabe d'eau douce de la Jamaïque, n'hébergent pas de Lagenophrys (Corliss et Brough, 1965).

\section{C) L'identité des Epistylis des Balanus de New-York.}

Il ne semble pas douteux que l'Epistylis de Balanus balanoides soit E. horizontalis Chatton (1936). Certes, les dimensions indiquées par Chatton sont sensiblement différentes de celles que nous avons relevées; il indique une taille moyenne de $55 \mu$ à $50 \mu$ pour le sédentaire et $75 \mu \times 40 \mu \times 20 \mu$ pour le migrateur, mais ce chercheur a opéré sur le vivant, alors que toutes nos mensurations ont porté sur des animaux soit simplement fixés, soit fixés et inclus à la paraffine; les différences de taille semblent donc 
pouvoir être rapportées à la déshydratation provoquée par la fixation et l'inclusion à la paraffine.

L'Epistylis balanorum vu, par Merejkowsky (1877), chez Balanus balanoides est très différent de l'Epistylis que nous avons trouvé chez le même hôte, ne serait-ce que par sa taille : $100 \mu$ de long et d'ailleurs Precht (1935) considère que l'Epistylis vu par Méréjkowsky est en réalité Myoschiton balanorum (Precht, 1935).

Les comparaisons de nos Epistylis avec les autres espèces du genre décrites depuis la fin du siècle dernier, n'auraient guère de portée puisque la plupart des Epistylis connus n'ont pas été étudiés à l'aide d'imprégnation argentique. Seuls les Epistylis étudiés récemment par Lom (1964): E. digitalis, E. lacustris, E. lwoffi peuvent leur être comparés. Or, il suffit de se rapporter au travail cité pour se persuader que les infraciliatures buccales, le nombre de tours des polycinétie et haplocinétie, soit sur le disque, soit dans le tube infundibulaire, la topographie des peniculus, l'extension de la strie germinale sont différentes.

\section{Bibliographie}

AвоNYI (A.), 1928. - Ueber die Epizoen der Orchestia cavimana. Arb. Ungar. biol. Forsch. Inst., 2, 5-23.

ANDRÉ (E.), 1910. - Sur quelques infusoires marins parasites et commensaux. Rev. Suisse Zool., 18, 173-187.

ARVY (L.) et LACOMBE (D.), 1968. - Activités enzymatiques traceuses dans \& l'appareil cémentaire » des Balanidae (Crustacea, Cirripedia). C.R. Acad. Sci., 267, D, 13261328,1 pl. h. t.

ARVY (L.) et LIGUORI (V.-R.), 1968. - Studies on the biology of barnacles : differences in muscular cytochromoxydase activity histochemically detectable in some Balanidae. Amer. Zool., 8, $\mathrm{n}^{\circ}$ 4, 383.

ARVy (L.), LACombe (D.) et Shimony (T.), 1968. - Alkaline phosphatase activity histochemically detectable in the cement apparatus of the Balanidae (Crustacea Cirripedia). Amer. Zool., 8, $\mathrm{N}^{\circ} 4,384$.

- et Nigrelli (R. F.), 1969. - Studies on the biology of barnacles: parasites of Balanus eburneus and $B$. balanoides from New York Harbor and a review of the parasites and diseases of other Cirripedia. Zoologica, 54.

AwerinZew (S. W.), 1936. - Zur biologie des Infusors Lagenophrys. Arch. f. Protistenk, $87,131-141$.

BIEgel (M.), 1954. - Beitrag zur Peritrichenfäuna der Umgebung Erlanger. Arch. für Protistenk., 100, 153-182.

BoNNEFOY (Fr.) et MARChal (J.), 1942. - Contribution à l'étude de quelques formes épizoïques des isopodes terrestres du N.-E. de la France. Annal. Parasit., 19, 151-155.

Butschli (O.), 1887. - Ciliata, in: «Brown's Klassen und Ordnungen des Thier-Reichs., 1 (3), p. $1228-1820$. 
Chatton (E.) et Lwoff (A.), 1930. - Imprégnation par diffusion argentique de l'infraciliature des ciliés marins et d'eau douce, après fixation cytologique et sans dessiccation. C.R. Soc. Biol., 104, 834.

- (1930). - The asymmetrical motile stage of an Epistylis and the question of the so-called longitudinal division of the Peritrichous Infusoria. The Collecting Net., 5, p. 372.

- 1936. - Les migrateurs horizontalement polarisés de certains Péritriches. De leur signification. Mém. Musée Roy. Hist. nat. Belgique, $2^{\circ}$ série, $\mathrm{n}^{\circ} 3,913-940,42$ fig.

— et Villeneuve (S.), 1937. - La division de la bouche et la formation du péristome chez les Péritriches (Cyclochaeta astropectinis, n. sp.). Leur continuité génétique immédiate. C.R. Acad. Sci., 204, 538.

Corliss (J. O.) et Brough (I. M.), 1965. - A new species of Lagenophrys (Ciliata: Peritrichida) from the jamaican crab Metopaulias depressus. Trans. amer. micr. soc., $84,73-80$.

Couch (J. A.), 1967. - A new species of Lagenophrys (Ciliatea: Peritrichida: Lagenophryidae) from a marine crab Callinectes sapidus. Trans. amer. micr. Soc., 86, $204-$ 211.

CuÉNot (L.), 1891. - Infusoires commensaux des ligies, patelles et arénicoles. Rev. biol. Nord France, 4, 81-89.

Dogiel (V.) et Furssenko (A.), 1920. - Neue ektoparasitische Infusorien von Landisopoden. Trav. Soc. nat. Petrograd., 51, 147-158.

Dragesco (J.), 1962. - L'orientation actuelle de la systématique des Ciliés et la technique d'imprégnation au protéinate d'argent. Bull. Micr. appl., 11, 49-58.

EhrenBerg (C. G.), 1838. - Die Infusionsthierchen als vollkommene Organismen. Leipzig, $612 \mathrm{p}$.

FAURÉ-Frémiet (E.), 1948. - Développement allométrique des constituants du pédoncule chez «Epistylis capitulum», n. sp. et «Opercularia cupulata», n. sp. Anais Acad. Brasil Ci., 20, $\mathrm{n}^{\circ}$ 2, 95-102.

FENCHEL (T.), 1965. - Ciliates from Scandinavian Molluscs. Ophelia, 2, 71-174.

-, 1965. - On the ciliate fauna associated with the marine species of the amphipod genus Gammarus J. G. Fabricius. Ophelia, 2, 281-303.

Hamilton (J. M.), 1952. - Studies on loricate Ciliophora. Cothurnia variabilis Kellicott. Tr. amer. micr. Soc., 71, 382-392.

Haswell (W. A.), 1901. - Note on the fauna of the gill cavities of fresh-water crayfishes. Rep. of the 8th Meet Australasian Assoc. Adv. Sci., Assoc. publ. Melbourne, 235-237.

Imamura (T.), 1940. - Two species of Lagenophrys from Sapporo. Annot. Zool. jap., 19, 267-270.

JANKowsKı (A. W.), 1967. - Ciliés dans la cavité palléale des Balanus de la mer de Barents. Parasitology, Acad. Sci. U.R.S.S., 1, 82-93.

KAHL (A.), 1934. - Ciliata ectocommensalia et parasitica, in: Grimpe (G.) et Wagler (E.). Die Tierwelt der Nord und Ostsee, 26, 147-183.

-, 1935. - Peritricha und Chonotricha, in: Dahl's (F.). Die Tierwelt Deutschlands, part 30, 1-886. 
KANE (J. R.), 1965. - The genus Lagenophrys Stein, 1852 (Ciliata, Peritricha) on Australasian Parastacidae. J. of Protozool, 12, 109-122.

Kellicotr (D. S.), 1883. - On some infusoria found on the crayfish. Proc. Amer. Soc. Micros., 6, 105-111.

-, 1894. - On certain marine infusoria observed at Woods-Hole, Mass. (Abstr.). 2nd Ann. Rep. Ohio St. Acad. Sci., 10-11.

KosLoff (E. N.), 1960. - Morphological studies on holotrichous ciliates of the family Hysterocinetidae. J. of Protozool., 7, 17-19.

Lóm (J.), 1961. - On the buccal apparatus of Peritrichous ciliates. Prog. in Protozool. first intern. Conf. Protozool., Prague, 91-95.

-, 1964. - The morphology and morphogenesis of the buccal ciliary organelles in some Peritrichous Ciliates. Arch. Protistenk., 107, 131-162.

Matrhes (D.), 1950. - Die Kiemenfauna unserer Landasseln. Zool. Jahrb., 78, 573-640.

—, 1950. - Beitrag zur Peritrichenfauna der Umgebung Erlangens. Zool. Jahrb., 79, 437.

-, 1955. - Neues zur Kiemenfauna der Landispoden. Ver Deutsch Zool. Ges. Erlangen.

—, 1955. - Die Herkunft isopodobionter Ciliaten im Lichte zweier neuer Funde. Arch. für Protistenk., 100, 331-350.

Mavrodiadi (P.), 1923. - « Kosoe» delenie u infusorii. Pratsy Belaruskaya dziarzk. univ. Mensku., 166, 4-5.

MerejkowsKy (K.), 1877. - Etudes sur les Protozoaires du nord de la Russie. Trav. Soc. natural St. Petersbourg, 7, 203-385.

Mouchet (S.), 1932. - Notes sur la biologie du galathéide Aeglea laevis (Latr.). Bull. Soc. Zool. Fr., 57, 316-340.

Mouchet-Bennati (S.), 1932. - Sur la branchie d'Aeglea laevis (Latreille) et son parasite Lagenophrys aeglea nov. sp. C.R. Soc. Biol., 109-148.

Nemec (B.), 1895. - O ectoparasitech Ligidia. Sitzber. k. Böhm. Ges. Wiss., 1 (32), 1-13, 1 pl. h.-t.

Nenninger (U.), 1948. - Die Peritrichen den Umgebung von Erlangen mit besonderer Berücksichtigung ihrer Wirtsspezifität. Zool. Jahrb., 77, 169-266.

NIE (D.) et Ho (Y. L.), 1943. - Notes on some epizoic infusoria from the fresh-water shrimp Palaemon nipponensis. Sinensia, 14, 143-149.

Norrot-Timothée (C.) et Lóm (J.), 1965. - L’ultrastructure de l'aplocinétie des Ciliés Péritriches. Comparaison avec la membrane ondulante des Hyménostomes. Protistologica, $1,33-39$.

Plate (L.), 1886. - Untersuchungen einiger an den Kiemenblättern des Gammarus pulex lebenden Ektoparasiten. Zeit. f. Wiss. Zool., 43, 175-241.

Precht (H.), 1935. - Epizoen der Kieler Bucht. Nova Acta Leopoldina, 3 (15), 405-474.

RAABE (Z.), 1947. - Drogi przystosowan morfologicznych do zycia posoeytnego wsrod wymoczkow. Ann. Univ. Mariae Curie Sklodowska, sect. C, 2, 299-411.

RÉmY (P.), 1928. - Balladora marcelli n. sp. Vorticellide commensal du Crustacé isopode terrestre Trichoniscus (Spiloniscus) provisorius Racovitza. Ann. Parasitol., 4, 19-30.

SHOMAY (D.), 1954. - The natural history of Lagenophrys labiata Stokes (Ciliata, Peritricha). J. of Protozool., 1, suppl., p. 9, abstr. $\mathrm{n}^{\circ} 43$. 
-, 1954. - The structure and life history of Lagenophrys labiata Stokes (Ciliata, Peritrichida). J. of Protozool., 1, suppl., abstr. $\mathrm{n}^{\circ} 10$.

Sommer (G.), 1951. - Die peritrichen Ciliaten des Grossen Plöner Sees. Archiv. für Hydrobiol., 44, 349-440.

Stammer (H.J.), 1948. - Eine neue eigenartige entoparasitische Peritriche Operculariella parasitica ng., n. sp. Zool. Jahrb., 77, 163-168.

STEIN (F.), 1851. - Neue Beiträge zur Kenntnis der Infusionsthiere. Zeit. für wis. Zool., 3, 485-492 et 499-506.

—, 1854. - Die Infusionsthiere auf ihre Entwicklungsgeschichte. Engelmann édit., Leipzig.

Stiller (J.) et Stevčic (Z.), 1967. - Epibionten der Meeresspinne Maja squinado (Herbst) nebst Beschreibung drei neuer Entokommensaler Peritrichenarten. Thalassia Jugoslavica, 3, 161-172.

Stokes (A. C.), 1887. - Notices of new fresh-water infusoria. Proc. amer. philos. Soc., 24, 244-255, 1 pl. h.-t.

SwarcZewsky (B.), 1928. - Beobachtungen über Spirochona elegans n. sp. Arch. f. Protistenk., 61, 185-222.

Tuffrau (M.), 1967. - Perfectionnement et pratique de la technique d'imprégnation au protargol des Infusoires ciliés. Protistologica, 3, 91-98.

Uвisch (M.), 1913. - Ein Beitrag zur Kenntnis des Gattung Lagenophrys. Arch. für Protistenk., 29, 37-77.

Wallengren (B. H.), 1900. - Ubersicht von der Gattung Lagenophrys Stein. Biol. Zentralb., $20,358-363$. 